\title{
Speed of a von Kármán point vortex street in a weakly compressible fluid
}

\author{
Darren G. Crowdy* and Vikas S. Krishnamurthy \\ Department of Mathematics, Imperial College London, 180 Queen's Gate, London SW7 2AZ, United Kingdom
}

(Received 9 June 2017; published 3 November 2017)

\begin{abstract}
Analytical expressions are obtained for the change in speed of translation of the von Kármán point vortex streets of given aspect ratios due to the effects of weak compressibility in subsonic flow of an isentropic fluid. We also clarify the nature of the force-free condition on a weakly compressible point vortex in equilibrium. For staggered streets, it is found that the speed of a compressible point vortex street can both increase and decrease relative to its incompressible counterpart of the same aspect ratio. Compressibility increases the speeds of streets with aspect ratios less than the critical value of 0.38187 , at which no change in speed occurs to first order in the (squared) Mach number. In particular, the compressible counterpart to the neutrally stable incompressible point vortex street of aspect ratio 0.28056 is found to propagate with increased speed. Streets with aspect ratios larger than 0.38187 slow down under the effects of compressibility, with the slowdown becoming maximal at a street aspect ratio of 0.52630 . On the other hand, the speed of unstaggered streets always increases, with the first-order correction in speed relative to its incompressible value increasing maximally at aspect ratios around $\kappa=0.36216$.
\end{abstract}

DOI: 10.1103/PhysRevFluids.2.114701

\section{INTRODUCTION}

The study of incompressible vortex dynamics is a vital subfield of fluid mechanics, especially important for the study of wakes [1], and it plays a crucial role in aerodynamics, geophysics, astrophysics, turbulence theory [2], and biofluids [3-5]. A surprising circumstance, remarked upon by previous authors [6], is that the study of compressible vortex dynamics has received markedly less attention even though gaining an understanding of compressible vortex flows is of fundamental interest for compressible wakes [7,8] and the theory of vortex sound and aeroacoustics [9-11]. There have been a few fundamental studies of compressible vortices [12-15], but many basic theoretical questions remain to be answered. There is, by now, evidence in the literature [16-20] of the existence of continuous families of shock-free transonic compressible flows with embedded vortices. The present paper aims to add to this small list of existing results.

For inviscid flows, a paradigmatic model of vortex wakes is the von Kármán vortex street in which the centers of vorticity shed in the wake of a bluff body are modeled by point vortices in an incompressible fluid [1,3,4,21,22]. This model and its various inviscid desingularizations have been well studied in the incompressible case, in particular with respect to their stability properties [23-26]. The compressible counterpart of the von Kármán vortex street, in which the same steadily translating vortex structures comprising two parallel vortex rows now exist in a compressible fluid, appears to have received little previous attention. A likely reason for this is the well-known theoretical obstruction to defining a point vortex singularity in a compressible fluid [27-29]: The fluid pressure becomes infinite as the point vortex location is approached, leading to physically unrealistic increases in density in a compressible fluid, where (for example, for an isentropic fluid) pressure and density are directly related. Yet this obstruction can be remedied by introducing a cutoff in a small region near the singularity in which a different model of the vortex core is introduced; previous authors have considered a thin core containing stagnant constant-pressure fluid [16], a "hollow vortex" core [30], or a light cylinder of small radius drifting along with the fluid $[17,18]$.

*d.crowdy@imperial.ac.uk 
In contrast to compressible vortex streets, the simpler situation of a cotraveling compressible vortex pair has already been investigated. Moore and Pullin [16] carried out a numerical study of it and also included a perturbation analysis of small-cored vortices for small Mach numbers (a perturbation expansion in small Mach numbers is called a Rayleigh-Jansen analysis [27-29]). Leppington [18] returned to the Rayleigh-Jansen analysis of small-cored vortices of Moore and Pullin [16] to point out an oversight and concluded that there is no change in the speed of a compressible vortex pair to first order in the (squared) Mach number. Leppington describes an approach via matched asymptotics to separate an outer far-field region where a wavelike flow might be expected to dominate, an intermediate region (away from the vortex) where a Rayleigh-Jansen expansion of the incompressible flow is a good description, and an inner core region where an equation first written down by Taylor [31] pertains. Leppington's focus is on finding the Rayleigh-Jansen expansion in the intermediate region, although he also discusses matching to both the far-field flow and the inner core region to complete a global asymptotic description of the flow.

A significant fact evident from Leppington's analysis is that a determination of the change in the speed of travel of the point vortex pair can be made purely from an analysis of the intermediate region [18]. All that is required is a Prandtl-Glauert-type assumption that compressibility effects are weak in the far field, an assumption on the general behavior of the near-field velocity, and a physical requirement that the compressible vortex remains free of net force. With the expectation that the same features will hold for a weakly compressible steadily translating vortex street, this paper addresses the basic theoretical question of how weak compressibility affects the speed of von Kármán point vortex streets of given aspect ratios. We show that, for staggered streets, if the speed $U$ of the compressible street is written for small Mach number $M=U_{0} / c_{s}$, where $c_{s}$ is the speed of sound in quiescent fluid, as the expansion

$$
U=U_{0}+M^{2} U_{1}+o\left(M^{2}\right), \quad U_{0}=\frac{\Gamma}{2 L} \tanh (\pi \kappa)
$$

then

$$
\frac{U_{1}}{U_{0}}=\frac{1}{2}\left[\operatorname{cosech}^{2}(\pi \kappa)-2 \pi \kappa \operatorname{cosech}(2 \pi \kappa)\right],
$$

where $\kappa$ is the aspect ratio of the staggered street (defined later). $U_{0}$ is the speed of propagation of the incompressible street. The analogous formula for unstaggered streets is also determined, and recorded in Eq. (61) of Sec. V.

The results (2) and (61) are direct generalizations of Leppington's analysis for the vortex pair [18]. Indeed, to elucidate our approach, in Sec. III we first retrieve Leppington's analysis for the compressible point vortex pair and, in Sec. IV, extend this analysis to the staggered von Kármán point vortex street. The analogous results for unstaggered streets are given in Sec. V. We do not consider here the matter of matching to any far field, or to the inner core region, since the analysis is expected to closely follow that sketched out by Leppington [18] for the vortex pair.

While precise details of the core structure do not affect the speed of the compressible vortex pair, that speed is nevertheless determined by a condition that the vortices are force-free. For two-dimensional point vortices in incompressible fluids, this force-free condition is known to be equivalent to setting the regular part of the fluid velocity in the vicinity of the vortex (sometimes referred to as the "non-self-induced velocity") to zero in the rest frame of the vortex. In his monograph, Saffman [3] remarks on the fact that this condition is often attributed to being a direct consequence of the Helmholtz laws of vortex motion - that vortex lines move with the fluid-but he points out that a more careful derivation is warranted owing to the singular nature of the point vortex flow. Llewellyn Smith [32] has discussed the matter of determining singularity motion in potential flow in some detail.

Leppington [18] uses an analogous non-self-induced velocity condition in his compressible flow analysis, quoting the fact that vortex lines move with the fluid, and he simply sets a uniform irrotational flow contribution in a local expansion of the first-order velocity potential to zero. 
No justification is offered as to why this remains the relevant condition when the fluid becomes compressible, a situation rendered even more unsatisfactory by the presence of several singular local contributions to the first-order correction to the velocity field that are directly associated with the fluid compressibility and which are ignored without explanation. A closely related matter had been considered earlier by Barsony-Nagy [33], who was interested in the extension of the Blasius force theorem [34] for weakly compressible flows and who derived integral expressions for the first-order correction (in Mach number) to the force on a solid body in steady subsonic flows. For small-cored vortices (where one can imagine "cutting off" the near-field singularity having close-to-circular streamlines by replacing the core with a density-matched circular cylinder as suggested by Leppington [18]), it turns out that the condition derived by Barsony-Nagy [33] gives the same result as the condition imposed by Leppington [18]. Leppington [18] does not mention the work of Barsony-Nagy [33] but he does refer to another study by Barsony-Nagy et al. [17] on the compressible Föppl problem of a point vortex wake behind a cylinder in uniform flow, where the authors employed the force-free condition derived by Barsony-Nagy in Ref. [33].

Even so, the connection between the force-free conditions imposed by Leppington [18] and by Barsony-Nagy et al. [17] is far from evident and it is the view of the present authors that the origin of this condition should not be glossed over, especially since it is this force-free condition that replaces the need for any analysis of the inner core region. Moreover, Leppington [18] assigns the source of the error in the earlier Rayleigh-Jansen analysis of Moore and Pullin [16] to their failure to impose any such force-free condition. Given this checkered history and the delicate nature of the matter, we include in an appendix our own derivation of this force-free condition for weakly compressible point vortices in equilibrium.

\section{THE IMAI-LAMLA METHOD}

To perform a Rayleigh-Jansen perturbation analysis for small Mach numbers about a given incompressible flow equilibrium, two possible approaches are available [27-29]. One method utilizes a hodograph formulation and the Chaplygin equation for the velocity potential [18,19]; an alternative method relies on a complex variable formulation of the equations of motion. The latter method, which will be employed here, is often called the Imai-Lamla method [28,29,35-37] and appears to be the lesser known approach.

Let the two-dimensional flow take place in an $(x, y)$ plane and let the fluid velocity field be $(u, v)$. If the flow is incompressible, inviscid, and irrotational apart from the presence of isolated point vortices, a stream function $\psi(x, y)$ exists such that

$$
u=\frac{\partial \psi}{\partial y} \quad \text { and } \quad v=-\frac{\partial \psi}{\partial x}
$$

as well as a velocity potential function $\phi(x, y)$ satisfying

$$
u=\frac{\partial \phi}{\partial x} \quad \text { and } \quad v=\frac{\partial \phi}{\partial y} .
$$

The equalities between partial derivatives implied by Eqs. (3) and (4) constitute the Cauchy-Riemann equations [34] for the harmonic functions $\phi(x, y)$ and $\psi(x, y)$, and one proceeds by defining the complex potential

$$
f(z)=\phi+i \psi
$$

which is an analytic function of the complex variable $z=x+i y$.

One may continue to use complex variables when the flow is compressible, with the important difference that the complex potential is no longer an analytic function [28,37]. We consider the isentropic flow of an ideal gas [27], with density $v(x, y)$ and pressure $p(x, y)$, for which the pressuredensity relationship is

$$
p=k v^{\gamma},
$$


where $k$ is a constant and $\gamma>1$ is the ratio of specific heats of the fluid. A generalized form of Kelvin's circulation theorem pertains to such flows [3], from which it can be argued that an initially irrotational flow remains so. If the flow is irrotational, as we assume here away from isolated point vortices, a velocity potential $\phi(x, y)$ exists and is again related to the velocity field by (4). On the other hand, for a steady two-dimensional flow, a stream function $\psi(x, y)$ also exists but it is now related to the velocity field through the density field $v(x, y)$ :

$$
u=\frac{v_{0}}{v} \frac{\partial \psi}{\partial y} \quad \text { and } \quad v=-\frac{\nu_{0}}{v} \frac{\partial \psi}{\partial x}
$$

where $v_{0}$ is some constant reference density. Clearly, (4) and (7) together no longer constitute the Cauchy-Riemann equations for harmonic functions $\phi$ and $\psi$. Nevertheless, we continue to define a complex potential [29]

$$
f(z, \bar{z})=\phi+i \psi
$$

which is now a function of both $z$ and its conjugate variable $\bar{z}$. On noting that

$$
\frac{\partial}{\partial z}=\frac{1}{2}\left[\frac{\partial}{\partial x}-i \frac{\partial}{\partial y}\right], \quad \frac{\partial}{\partial \bar{z}}=\frac{1}{2}\left[\frac{\partial}{\partial x}+i \frac{\partial}{\partial y}\right],
$$

it is readily shown on combining (4) and (7) that

$$
2 \frac{\partial f}{\partial \bar{z}}=\left(1-\frac{v}{v_{0}}\right)(u+i v), \quad 2 \frac{\partial \bar{f}}{\partial z}=\left(1+\frac{v}{v_{0}}\right)(u+i v) .
$$

On equating expressions for $u+i v$ derived from the two equations in (10), we find

$$
\frac{\partial f}{\partial \bar{z}}=B(v) \frac{\partial \bar{f}}{\partial \bar{z}}, \quad \text { where } B(v)=\frac{1-v / v_{0}}{1+v / v_{0}} .
$$

The complex velocity field $\xi(z, \bar{z}) \equiv u-i v$ can be written either in terms of the complex potential as

$$
\xi(z, \bar{z})=u-i v=\frac{\partial \phi}{\partial x}-i \frac{\partial \phi}{\partial y}=\frac{\partial}{\partial z}(f+\bar{f})
$$

or, on use of (11), in terms of the complex potential and density as

$$
\xi(z, \bar{z})=\frac{\partial f}{\partial z}[1+B(v)] .
$$

The dynamics is governed by the Bernoulli equation for steady irrotational compressible flows which states that

$$
\frac{|\xi|^{2}}{2}+\int \frac{d p}{v}=\text { constant }
$$

On use of (6), we can write this as

$$
\frac{|\xi|^{2}}{2}+\frac{k \gamma \nu^{\gamma-1}}{\gamma-1}=\text { constant }=\frac{k \gamma \nu_{s}^{\gamma-1}}{\gamma-1},
$$

where $v_{s}$ is the density at a stagnation point where $|\xi|=0$. The speed of sound $c$ in the fluid is defined via

$$
c^{2}=\frac{d p}{d \nu}=\frac{\gamma p}{\nu}=\gamma k v^{\gamma-1}
$$

The speed of sound $c_{s}$ at a stagnation point in the fluid is

$$
c_{s}^{2}=\left.\frac{d p}{d v}\right|_{v_{s}}=\gamma k v_{s}^{\gamma-1}
$$


where $v_{s}$ denotes the density of quiescent fluid. We can use this to define the Mach number

$$
M=\frac{V_{0}}{c_{s}},
$$

where $V_{0}$ is some typical velocity associated with the flow. Different choices of $V_{0}$ will be made for the various flow scenarios considered later. It follows from the Bernoulli equation (15) that

$$
\frac{|\xi|^{2}}{2}+\frac{c_{s}^{2}}{\gamma-1}\left(\frac{v}{v_{s}}\right)^{\gamma-1}=\frac{c_{s}^{2}}{\gamma-1}
$$

which can be rearranged to give

$$
\left(\frac{v}{v_{s}}\right)^{\gamma-1}=1-\frac{\gamma-1}{2}|\xi|^{2} \frac{M^{2}}{V_{0}^{2}}
$$

where we have made use of (18).

In this study, we restrict attention to weakly compressible flows for which $M \ll 1$ so that the complex potential and the velocity field can be written as a Rayleigh-Jansen expansion [28] whose leading-order terms are given by the incompressible solution. Following Leppington [18], we assume that a subsonic global flow can be found. The Rayleigh-Jansen analysis proceeds by developing the complex potential and velocity as the regular expansions

$$
\begin{aligned}
& f(z, \bar{z})=f_{0}(z)+M^{2} f_{1}(z, \bar{z})+o\left(M^{2}\right), \\
& \xi(z, \bar{z})=\xi_{0}(z)+M^{2} \xi_{1}(z, \bar{z})+o\left(M^{2}\right),
\end{aligned}
$$

where $f_{0}(z)$ and $\xi_{0}(z)=d f_{0} / d z$ are determined by the incompressible solution. $f_{1}(z, \bar{z})$ and $\xi_{1}(z, \bar{z})$ are the first-order corrections due to compressibility and are to be computed. The so-called ImaiLamla formula provides [28,29,35-37] an expression for the complex potential $f_{1}(z, \bar{z})$ up to an unknown analytic function $G(z)$, viz.,

$$
f_{1}(z, \bar{z})=\frac{1}{4 V_{0}^{2}} \xi_{0}(z) \overline{I(z)}+G(z)
$$

where $I(z)$ is what we will refer to as the Imai-Lamla integral:

$$
I(z)=\int^{z} \xi_{0}(z)^{2} d z
$$

Formulas (23) and (24) are derived as follows. If $M \ll 1$, we can develop the following expansion of (20):

$$
\frac{v}{v_{s}}=1-M^{2} \frac{|\xi|^{2}}{2 V_{0}^{2}}+o\left(M^{2}\right)
$$

This is essentially an expansion of the Bernoulli equation. Without loss of generality, we can choose the reference density $v_{0}=v_{s}$ and it then follows from (11) and (25) that

$$
B(v)=M^{2} \frac{|\xi|^{2}}{4 V_{0}^{2}}+o\left(M^{2}\right), \quad \frac{\partial f}{\partial \bar{z}}=M^{2} \frac{|\xi|^{2}}{4 V_{0}^{2}} \frac{\partial \bar{f}}{\partial \bar{z}}+o\left(M^{2}\right)
$$

On substitution of the Rayleigh-Jansen expansions (21) and (22) into (26), we find

$$
\frac{\partial f_{0}}{\partial \bar{z}}+M^{2} \frac{\partial f_{1}}{\partial \bar{z}}=M^{2} \frac{\left|\xi_{0}\right|^{2}}{4 V_{0}^{2}} \frac{\partial \overline{f_{0}}}{\partial \bar{z}}+o\left(M^{2}\right)
$$


The leading-order term in this expansion merely confirms that the leading-order flow is irrotational. On equating the coefficients of $M^{2}$ in (27), and on integration with respect to $\bar{z}$, we arrive at the Imai-Lamla formula (23).

Once the function $G(z)$ in (23) has been identified, an expression for the velocity $\xi_{1}(z, \bar{z})$ may then be obtained by using the Rayleigh-Jansen expansion (21) and the Imai-Lamla formula (23) in (12):

$$
\xi_{1}(z, \bar{z})=\frac{1}{4 V_{0}^{2}}\left[\left(\xi_{0}(z)\right)^{2} \overline{\xi_{0}(z)}+\xi_{0}^{\prime}(z) \overline{I(z)}\right]+G^{\prime}(z) .
$$

The prime notation denotes a derivative with respect to $z$. It is clear that it is the matter of finding the relevant function $G(z)$ pertinent to a particular flow scenario that becomes the challenge within this Imai-Lamla approach.

\section{THE COMPRESSIBLE VORTEX PAIR}

We will apply the method just described to find the first-order correction to the speed of translation of a weakly compressible cotraveling point vortex pair. First, consider a pair of incompressible point vortices, of circulations $\pm \Gamma$, located at positions $\pm i a_{0}$ in a frame of reference cotravelling with the speed $U_{0}$ of steady translation of the vortex pair where

$$
U_{0}=\frac{\Gamma}{4 \pi a_{0}} .
$$

Deriving this expression for $U_{0}$, and the associated incompressible complex potential

$$
f_{0}(z)=-U_{0} z-\frac{i \Gamma}{2 \pi} \log \left(z-i a_{0}\right)+\frac{i \Gamma}{2 \pi} \log \left(z+i a_{0}\right)
$$

is a standard exercise in inviscid fluid mechanics [34]. The corresponding complex velocity $\xi_{0}=u-i v=d f_{0} / d z$ is given by

$$
\xi_{0}(z)=-U_{0}-\frac{i \Gamma}{2 \pi}\left[\frac{1}{z-i a_{0}}-\frac{1}{z+i a_{0}}\right] .
$$

We now seek a Rayleigh-Jansen expansion about this incompressible solution taking the typical velocity scale $V_{0}$ in the definition (18) of the Mach number to be $U_{0}$. The perturbed speed of the vortex pair is given by (1) where the objective is to determine $U_{1}$. From the Imai-Lamla formulation, the first-order correction to the complex potential is

$$
f_{1}(z, \bar{z})=\frac{\xi_{0}(z)}{4 U_{0}^{2}} \overline{I(z)}+G(z)
$$

where $I(z)$ is defined in (24) and the analytic function $G(z)$ has to be found. On use of (31), and after performing the integration, we find

$$
I(z)=\frac{\Gamma^{2}}{4 \pi^{2}}\left[\frac{1}{z-i a_{0}}+\frac{1}{z+i a_{0}}+\frac{z}{4 a_{0}^{2}}\right] .
$$

We choose the following form for $G(z)$ :

$$
G(z)=-\frac{\xi_{0}(z) I(z)}{4 U_{0}^{2}}+\tilde{G}(z),
$$

so that

$$
f_{1}(z, \bar{z})=\frac{\xi_{0}(z)}{4 U_{0}^{2}}[\overline{I(z)}-I(z)]+\tilde{G}(z) .
$$


With this choice, it is easy to verify from (28) that any change in the far-field velocity due to the effects of compressibility will derive from the contribution from $\tilde{G}(z)$. We therefore write

$$
\tilde{G}(z)=-U_{1} z+\frac{\mu}{z-i a_{0}}+\frac{\mu}{z+i a_{0}}+\frac{\lambda}{\left(z-i a_{0}\right)^{2}}-\frac{\lambda}{\left(z+i a_{0}\right)^{2}},
$$

where $\mu, \lambda \in \mathbb{C}$ are constants to be determined and $U_{1} \in \mathbb{R}$ is the required correction to the speed of the vortex pair. This ansatz has been chosen to ensure that the complex potential is odd under the transformation $z \mapsto-z$. Its chosen isolated poles, which appear mysterious at this stage, have been chosen on the basis of the considerations to follow.

We now let

$$
\epsilon=z-i a_{0}
$$

and perform a local analysis near $z=i a_{0}$ so we assume $|\epsilon| \ll 1$. After some algebra, we find

$$
\begin{aligned}
& \xi_{0}(z)=-\frac{i \Gamma}{2 \pi}\left[\frac{1}{\epsilon}-\frac{\epsilon}{4 a_{0}^{2}}-\frac{i \epsilon^{2}}{8 a_{0}^{3}}+o\left(\epsilon^{2}\right)\right], \quad I(z)=\frac{\Gamma^{2}}{4 \pi^{2}}\left[\frac{1}{\epsilon}-\frac{i}{4 a_{0}}+\frac{\epsilon}{2 a_{0}^{2}}+\frac{i \epsilon^{2}}{8 a_{0}^{3}}+o\left(\epsilon^{2}\right)\right], \\
& \tilde{G}(z)=\frac{\lambda}{\epsilon^{2}}+\frac{\mu}{\epsilon}-i U_{1} a_{0}-\frac{i \mu}{2 a_{0}}+\frac{\lambda}{4 a_{0}^{2}}+\epsilon\left[\frac{i \lambda}{4 a_{0}^{3}}+\frac{\mu}{4 a_{0}^{2}}-U_{1}\right]+o(\epsilon) .
\end{aligned}
$$

On substitution into (35), we arrive at

$$
\begin{aligned}
f_{1}(z, \bar{z})= & -\frac{i \Gamma a_{0}^{2}}{2 \pi}\left[\frac{1}{|\epsilon|^{2}}-\frac{1}{\epsilon^{2}}+\frac{i}{2 a_{0} \epsilon}-\frac{1}{4 a_{0}^{2}}+\frac{\bar{\epsilon}}{\epsilon} \frac{1}{2 a_{0}^{2}}-\frac{\epsilon}{\bar{\epsilon}} \frac{1}{4 a_{0}^{2}}-\frac{i \epsilon}{8 a_{0}^{3}}-\frac{i \bar{\epsilon}^{2}}{8 \epsilon a_{0}^{3}}-\frac{i \epsilon^{2}}{8 \bar{\epsilon} a_{0}^{3}}\right] \\
& +\frac{\lambda}{\epsilon^{2}}+\frac{\mu}{\epsilon}-i U_{1} a_{0}-\frac{i \mu}{2 a_{0}}+\frac{\lambda}{4 a_{0}^{2}}+\epsilon\left[\frac{i \lambda}{4 a_{0}^{3}}+\frac{\mu}{4 a_{0}^{2}}-U_{1}\right]+o(\epsilon) .
\end{aligned}
$$

We now impose the following three conditions to determine the modified flow correct to first order in $M^{2}$ : (a) We ensure, for purposes of matching to an inner region associated with a compressible vortex (as opposed to any higher order singularity type) [18], that the velocity potential $\phi_{1}=\operatorname{Re}\left[f_{1}\right]$ grows no more quickly that $\mathcal{O}(1 / \epsilon)$ as $\epsilon \rightarrow 0$; (b) furthermore, if we insist that the centroid of the compressible vortex coincides with that of the leading-order incompressible vortex, then even any contribution to $\phi_{1}$ that is of $\mathcal{O}(1 / \epsilon)$ must vanish (although other choices can be made here if the compressible vortex position is also perturbed as was necessary, for example, in the compressible Foppl problem considered by Barsony-Nagy et al. [17]); and (c) that the compressible vortex is force-free correct to first order in $M^{2}$. While the first and third conditions are necessary, the second condition results from our particular choice to keep the centroid of the perturbed vortex fixed and then to determine the resulting change in the speed of propagation (note that Leppington, in his analysis, chooses to retain the freedom to alter the centroid location [18]). It should be noted that the term proportional to $1 /|\epsilon|^{2}$ in $f_{1}$ is purely imaginary, and therefore does not contribute to the velocity potential $\phi$.

For a discussion of the matching criterion, the reader is referred to Leppington's paper [18] where he discusses this in detail and gives the equation first derived by Taylor [31] describing the velocity potential in the inner core region (see also Barsony-Nagy et al. [17]). We omit further discussion of the matching here since, as we have already emphasized, aside from providing the aforementioned growth criterion on the outer solution as the inner region is approached, no further details of the inner core region are needed to determine the speed modification of the vortex pair at first order in $M^{2}$.

On the other hand, the matter of how to impose the force-free condition is important. In the appendix, we establish that the first-order correction to the force on the vortex, where we expand the total force $X-\mathrm{i} Y$ on the vortex in an expansion of the form

$$
X-i Y=\left(X_{0}-i Y_{0}\right)+M^{2}\left(X_{1}-i Y_{1}\right)+o\left(M^{2}\right)
$$




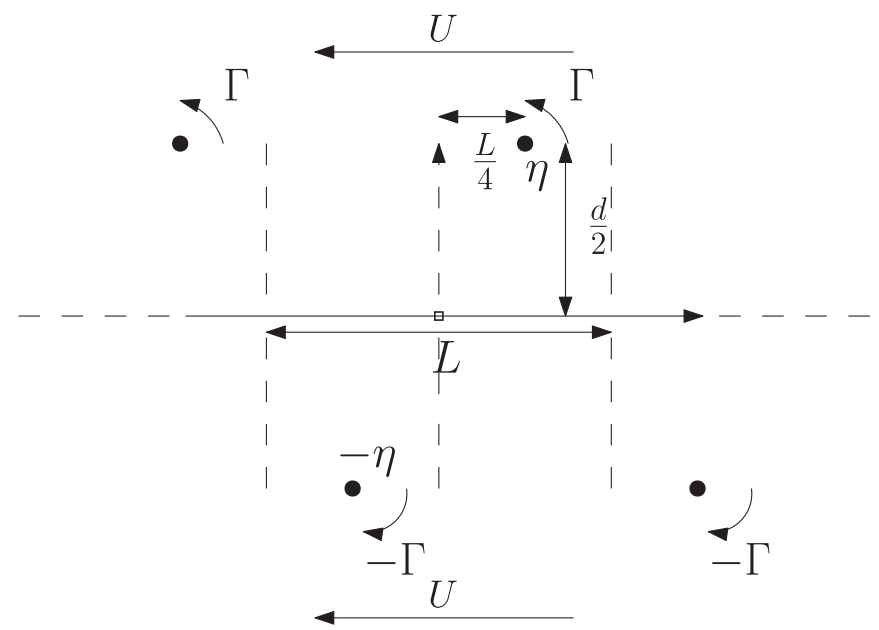

FIG. 1. A staggered periodic von Kármán point vortex street of aspect ratio $\kappa=d / L$ in a frame cotraveling with speed $U$. The challenge is to find the first-order change in the speed $U_{1}$ in (1) due to weak compressibility.

is given by

$$
X_{1}-i Y_{1}=\frac{i v_{s}}{4 U_{0}^{2}} \oint_{\mathcal{C}} d\left\{\frac{\left[\xi_{0}(z)\right]^{2}}{2} \overline{I(z)}\right\}+i v_{s} \oint_{\mathcal{C}} \xi_{0}(z) G^{\prime}(z) d z
$$

The Appendix demonstrates how setting this first-order force correction to zero is equivalent to setting to zero the term proportional to $\epsilon$ in the local expansion (39) of $f_{1}(z, \bar{z})$. Leppington [18] did precisely this in his Rayleigh-Jansen analysis of the point vortex pair but, in our view, without adequate explanation as to why it is equivalent to eliminating the first-order force correction on the vortex, a matter that becomes quite delicate for a compressible fluid, as evident from the treatment given in the Appendix.

To eliminate the terms in (39) proportional to $1 / \epsilon^{2}$ and $1 / \epsilon$, we must choose

$$
\lambda=-\frac{i \Gamma a_{0}^{2}}{2 \pi}, \quad \mu=-\frac{\Gamma a_{0}}{4 \pi} ;
$$

note that this choice of $\lambda$ cancels out all the second-order pole contribution to $G(z)$ (i.e., $G(z)$ has a removable second-order pole). Elimination of the coefficient of $\epsilon$ then leads to

$$
U_{1}=\frac{\mu}{4 a_{0}^{2}}+\frac{i \lambda}{4 a_{0}^{3}}-\frac{\Gamma}{16 \pi a_{0}}=0
$$

if the vortex positions are unchanged. In this way, we retrieve Leppington's result that, to first order in $M^{2}$, there is no change in the speed of a point vortex pair due to compressibility effects.

\section{COMPRESSIBLE STAGGERED POINT VORTEX STREET}

We now extend the foregoing analysis to the case of the staggered von Kármán point vortex street, as shown in Fig. 1. This analysis is new. The periodicity of the flow in this case must be properly accounted for, but the main steps in the construction are identical to that given in the previous section.

We focus on a period window, of length $L$, comprising a vortex of circulation $\Gamma$ at $\eta$ and a vortex of circulation $-\Gamma$ at $-\eta$ where

$$
\eta=\frac{L}{4}+\frac{i d}{2} .
$$


In a frame of reference cotranslating with the street at velocity $U_{0}$ where

$$
U_{0}=\frac{i \Gamma}{2 L} \cot \left(\frac{2 \pi \eta}{L}\right)=\frac{\Gamma}{2 L} \tanh \pi \kappa, \quad \kappa=\frac{d}{L},
$$

and where $\kappa$ is the street aspect ratio, then the complex potential is

$$
f_{0}(z)=-U_{0} z-\frac{i \Gamma}{2 \pi}\left\{\log \sin \left[\frac{\pi(z-\eta)}{L}\right]-\log \sin \left[\frac{\pi(z+\eta)}{L}\right]\right\}
$$

with corresponding complex velocity $\xi_{0}=u-\mathrm{i} v$ given by

$$
\xi_{0}(z)=-U_{0}-\frac{i \Gamma}{2 L}\left\{\cot \left[\frac{\pi(z-\eta)}{L}\right]-\cot \left[\frac{\pi(z+\eta)}{L}\right]\right\} .
$$

As in Sec. III we consider a Rayleigh-Jansen expansion about this incompressible solution taking the typical velocity scale $V_{0}$ in the definition (18) of the Mach number to be $U_{0}$. It must be noted that, with this choice, we assume that the aspect ratio of the street $\kappa$ remains bounded away from zero since, in that case, $U_{0} \rightarrow 0$ and, hence, $M \rightarrow 0$, and a different choice of Mach number would be appropriate in this case. This can easily be done a posteriori if required by a simple rescaling.

The perturbed speed of the vortex pair is given by (1) where $U_{1}$ must be determined. From the Imai-Lamla formulation, the first-order correction to the complex potential is

$$
f_{1}(z, \bar{z})=\frac{\xi_{0}(z)}{4 U_{0}^{2}} \overline{I(z)}+G(z)
$$

where $I(z)$ is defined in (24) and the function $G(z)$ has to be found. On use of (47), and after performing the integration, we find

$$
I(z)=U_{0}^{2} z+\frac{\Gamma^{2}}{4 \pi L}\left\{\cot \left[\frac{\pi(z-\eta)}{L}\right]+\cot \left[\frac{\pi(z+\eta)}{L}\right]\right\} .
$$

The function $G(z)$ is not required to be $L$ periodic, but it must be chosen to ensure that the associated velocity field is $L$ periodic. Indeed, for this purpose we must choose

$$
G(z)=-\frac{\xi_{0}(z)}{4 U_{0}^{2}} I(z)+\tilde{G}(z)
$$

so that

$$
f_{1}(z, \bar{z})=\frac{\xi_{0}(\zeta)}{4 U_{0}^{2}}[\overline{I(z)}-I(z)]+\tilde{G}(z)
$$

since it can now be verified that the first term is invariant as $z \mapsto z+L$ and where an additional $L$-periodic function $\tilde{G}(z)$ remains to be found. While it must be periodic, the latter is not necessarily analytic in the fluid. Indeed, motivated by the analysis in Sec. III, we choose

$$
\begin{aligned}
\tilde{G}(z)= & -U_{1} z+\mu \cot \left[\frac{\pi(z-\eta)}{L}\right]+\mu \cot \left[\frac{\pi(z+\eta)}{L}\right] \\
& +\lambda \operatorname{cosec}^{2}\left[\frac{\pi(z-\eta)}{L}\right]-\lambda \operatorname{cosec}^{2}\left[\frac{\pi(z+\eta)}{L}\right],
\end{aligned}
$$

where $\mu, \lambda \in \mathbb{C}$ are constants and where the form of this ansatz ensures that the potential is odd under the transformation $z \mapsto-z$ (recall that the $L$-periodic $\operatorname{cotangent}$ function $\cot (\pi z / L$ ) has a simple pole at $z=0$ while the $L$-periodic function $\operatorname{cosec}^{2}(\pi z / L)$ has a second-order pole there). 
Then,

$$
\begin{aligned}
f_{1}(z, \bar{z})= & \frac{\xi_{0}(z)}{4 U_{0}^{2}}[\overline{I(z)}-I(z)]+\mu \cot \left[\frac{\pi(z-\eta)}{L}\right]+\mu \cot \left[\frac{\pi(z+\eta)}{L}\right] \\
& +\lambda \operatorname{cosec}^{2}\left[\frac{\pi(z-\eta)}{L}\right]-\lambda \operatorname{cosec}^{2}\left[\frac{\pi(z+\eta)}{L}\right]-U_{1} z .
\end{aligned}
$$

To perform a local analysis near $\eta$, we now introduce the notation

$$
\epsilon=\frac{\pi}{L}(z-\eta), \quad \alpha=\cot \left(\frac{2 \pi \eta}{L}\right)
$$

We will need the expansions

$$
\begin{aligned}
& \cot \left[\frac{\pi(z-\eta)}{L}\right]=\frac{1}{\epsilon}-\frac{\epsilon}{3}+o(\epsilon), \quad \cot \left[\frac{\pi(z+\eta)}{L}\right]=\alpha-\epsilon\left(1+\alpha^{2}\right)+\epsilon^{2} \alpha\left(1+\alpha^{2}\right)+o\left(\epsilon^{2}\right), \\
& \operatorname{cosec}^{2}\left[\frac{\pi(z-\eta)}{L}\right]=\frac{1}{\epsilon^{2}}+\frac{1}{3}+o(1), \quad \operatorname{cosec}^{2}\left[\frac{\pi(z+\eta)}{L}\right]=\left(1+\alpha^{2}\right)-2 \epsilon \alpha\left(1+\alpha^{2}\right)+o(\epsilon) .
\end{aligned}
$$

After some manipulations, it can be shown that

$$
\begin{aligned}
I(z) & =-\frac{U_{0}^{2} L}{\pi \alpha^{2}}\left[\frac{1}{\epsilon}+\left(\alpha-\frac{\pi \eta \alpha^{2}}{L}\right)-2 \epsilon\left(\alpha^{2}+\frac{2}{3}\right)+\epsilon^{2} \alpha\left(1+\alpha^{2}\right)+o\left(\epsilon^{2}\right)\right], \\
\xi_{0}(z) & =-\frac{i \Gamma}{2 L}\left[\frac{1}{\epsilon}+\epsilon\left(\frac{2}{3}+\alpha^{2}\right)-\epsilon^{2} \alpha\left(1+\alpha^{2}\right)+o\left(\epsilon^{2}\right)\right] .
\end{aligned}
$$

It follows that

$$
\begin{aligned}
f_{1}(z, \bar{z})= & \frac{i \Gamma}{8 \pi \alpha^{2}} \frac{1}{|\epsilon|^{2}}+\frac{1}{\epsilon^{2}}\left[\lambda-\frac{i \Gamma}{8 \pi \alpha^{2}}\right]+\frac{1}{\epsilon}\left\{\mu-\frac{i \Gamma}{8 \pi \alpha^{2}}\left[2 \alpha-\frac{\pi \alpha^{2}(\eta-\bar{\eta})}{L}\right]\right\} \\
& +\frac{i \Gamma}{8 \pi \alpha^{2}}\left(\frac{2}{3}+\alpha^{2}\right)-U_{1} \eta+\mu \alpha-\lambda\left(1+\alpha^{2}\right)+\frac{i \Gamma}{8 \pi \alpha^{2}}\left[-2\left(\frac{2}{3}+\alpha^{2}\right) \frac{\bar{\epsilon}}{\epsilon}+\left(\frac{2}{3}+\alpha^{2}\right) \frac{\epsilon}{\bar{\epsilon}}\right] \\
& +\epsilon\left\{-\frac{U_{1} L}{\pi}-\mu\left(\frac{4}{3}+\alpha^{2}\right)+2 \alpha \lambda\left(1+\alpha^{2}\right)-\frac{i \Gamma}{8 \pi \alpha^{2}}\left(\frac{2}{3}+\alpha^{2}\right)\left[2 \alpha-\frac{\pi \alpha^{2}(\eta-\bar{\eta})}{L}\right]\right\} \\
& -\frac{i \Gamma\left(1+\alpha^{2}\right)}{8 \pi \alpha}\left(\frac{\bar{\epsilon}^{2}}{\epsilon}+\frac{\epsilon^{2}}{\bar{\epsilon}}\right)+o(\epsilon) .
\end{aligned}
$$

The purely imaginary term proportional to $1 /|\epsilon|^{2}$ does not affect the velocity potential, but to eliminate the second-order pole of $f_{1}(z, \bar{z})$ at $z=\eta$ we let

$$
\lambda=\frac{i \Gamma}{8 \pi \alpha^{2}} .
$$

This cancels off the $1 / \epsilon^{2}$ term (notice, again, that this choice makes the second-order pole of $G(z)$ removable). For a compressible vortex street with the same centroid location at $\eta$ as the unperturbed incompressible case, we must also remove the first-order pole in $f_{1}$ at $\eta$, leading to

$$
\mu=\frac{i \Gamma}{8 \pi \alpha^{2}}\left[2 \alpha-\frac{\pi \alpha^{2}(\eta-\bar{\eta})}{L}\right],
$$

which kills off the $1 / \epsilon$ term. Once again, from (47) and (49), we see that the quantity $\xi_{0}^{2}(z) \overline{I(z)}$ is single-valued around each vortex so, as shown in the appendix, to enforce the force-free condition 


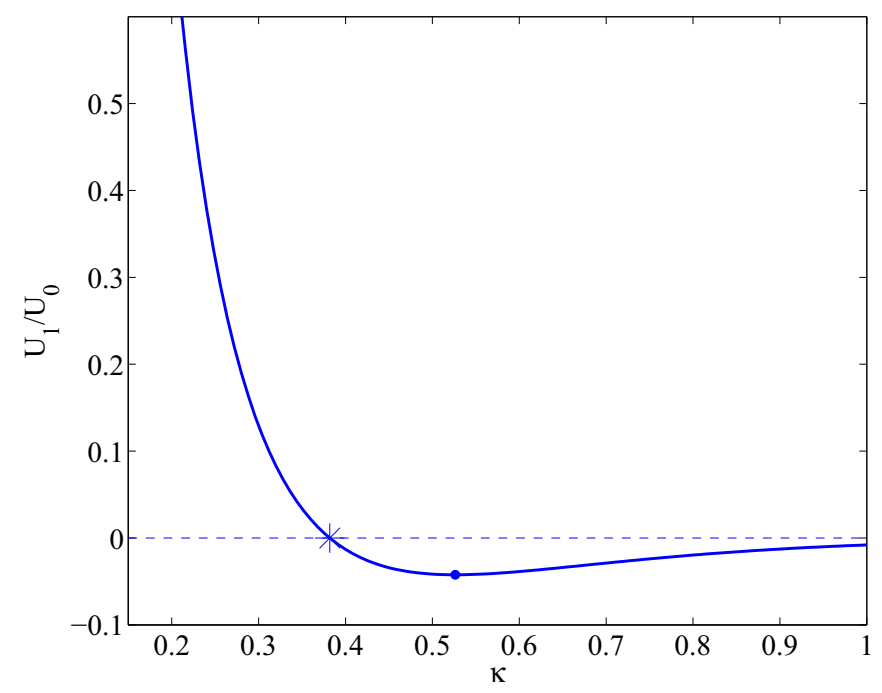

FIG. 2. The relative first-order correction $U_{1} / U_{0}$ in the speed of a weakly compressible staggered von Kármán point vortex street as a function of its aspect ratio. The graph shows that $U_{1} / U_{0}$ vanishes at $\kappa=0.38187$ and exhibits a local minimum at $\kappa=0.52630$.

we eliminate the term in the expression in (57) for $f_{1}(z, \bar{z})$ that is proportional to $\epsilon$. This leads, after some algebra, to

$$
U_{1}=-\frac{i \Gamma}{4 L}\left[\frac{1}{\alpha}+\alpha-\frac{\pi}{L}\left(1+\alpha^{2}\right)(\eta-\bar{\eta})\right] .
$$

The relative change in speed is then found, after further algebra and use of (45), to be given by formula (2).

Figure 2 shows a graph of $U_{1} / U_{0}$ as given by (2) as a function of street aspect ratio $\kappa$. Several features are of interest. First, as $\kappa \rightarrow 0$, we notice that $U_{1} / U_{0} \rightarrow \infty$, a singularity that does not concern us because we recognized earlier that $\kappa$ must remain bounded away from zero (the situation of an alternating vortex row) since that case corresponds to our expansion parameter $M \rightarrow 0$. For the physically interesting case of $\kappa=0.28056$ we find $U_{1} / U_{0}=0.18835$, indicating that the speed of such streets increases as compressibility comes into play. At the critical aspect ratio of $\kappa=0.38187$, the first-order flow correction vanishes, meaning that compressibility does not affect the speed of the street at first order in $M^{2}$, a feature that Leppington [18] found to be true of a weakly compressible cotraveling vortex pair as confirmed in Sec. III. On the other hand, for larger aspect ratios $\kappa>0.38187$, we find that $U_{1} / U_{0}$ is negative, so compressibility slows down large aspect ratio vortex streets, with a local minimum of $U_{1} / U_{0}=-0.04356$ reached at $\kappa=0.52630$. Finally, as the aspect ratio gets large, so that the effect of the stagger between the two vortex rows decreases, we find that $U_{1} / U_{0} \rightarrow 0$.

\section{COMPRESSIBLE UNSTAGGERED POINT VORTEX STREET}

The analysis of the previous section is readily extended to unstaggered streets, having the geometry depicted in Fig. 3, with the result

$$
\frac{U_{1}}{U_{0}}=\frac{1}{2}\left[2 \pi \kappa \operatorname{cosech}(2 \pi \kappa)-\operatorname{sech}^{2}(\pi \kappa)\right], \quad U_{0}=\frac{\Gamma}{2 L} \operatorname{coth}(\pi \kappa),
$$

where $\kappa=d / L$ is the aspect ratio of the unstaggered street. As $\kappa \rightarrow 0$, the effect of periodicity should become negligible and the result should tend to the value calculated earlier for an isolated 


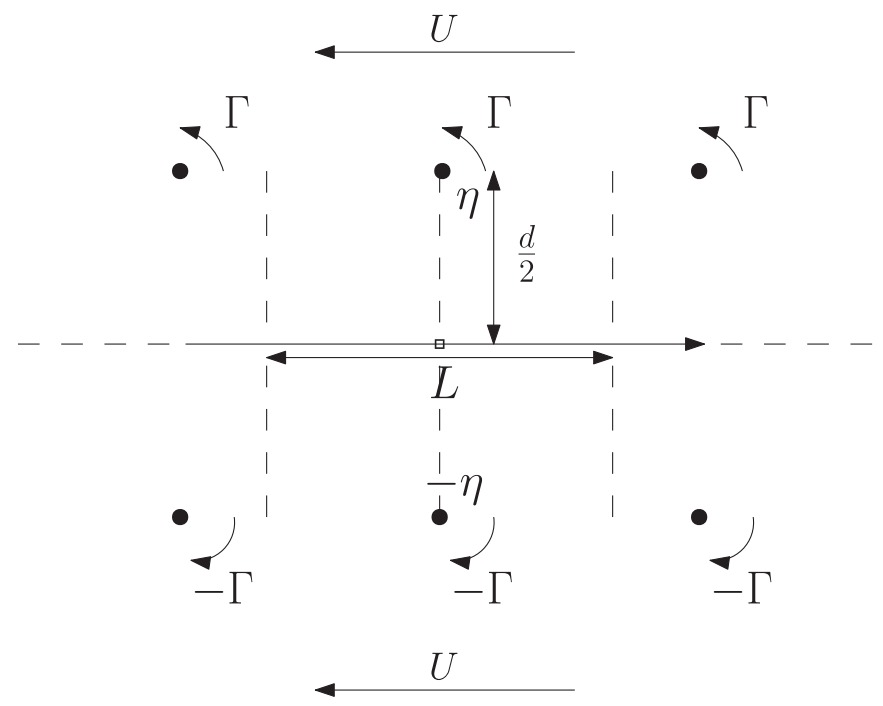

FIG. 3. A unstaggered periodic von Kármán point vortex street of aspect ratio $\kappa=d / L$ in a frame cotraveling with speed $U$.

point vortex pair in Sec. III. Indeed, on taking a limit of (61) as $\kappa \rightarrow 0$ it is found that $U_{1} / U_{0} \rightarrow 0$, and this provides a corroborative check on the analysis. Figure 4 shows a graph of (61) as a function of aspect ratio $\kappa$. It is always positive, so weakly compressible unstaggered streets always speed up relative to their incompressible counterparts. Moreover, a local maximum increase in this relative first-order correction to the speed is found at $\kappa=0.36216$.

It is interesting that the aspect ratio $\kappa=0.36216$ at which the speed of the unstaggered street is most affected by weak compressibility is close to the value $\kappa=0.38187$ at which the speed of the staggered street is unaffected. Although their studies had nothing to do with compressibility and pertain to finite-area vortices, it is intriguing to note that both Saffman and Schatzmann [38] (streets

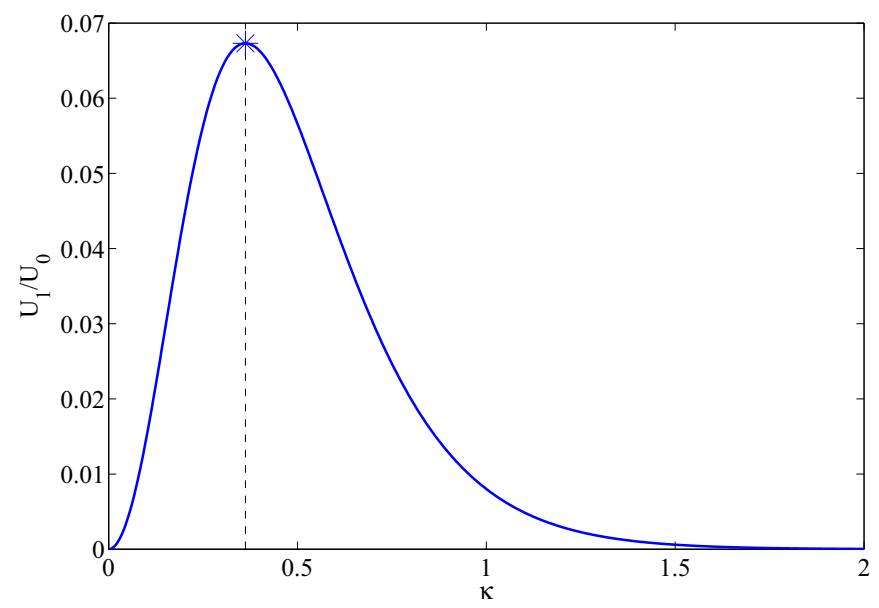

FIG. 4. The relative first-order correction $U_{1} / U_{0}$ in the speed of a weakly compressible unstaggered von Kármán point vortex street as a function of its aspect ratio. The graph shows a local maximum of $U_{1} / U_{0}=$ 0.06731 at $\kappa=0.36216$. 
of vortex patches) and Crowdy and Green [39] (streets of hollow vortices) also find that aspect ratios around 0.36 have special significance for the class of incompressible finite-area vortex streets found there.

\section{DISCUSSION}

This paper has contributed to the small but growing body of theoretical work on compressible vortex dynamics and, in particular, to the study of smooth transonic flows with embedded vortices.

An explicit formula (2) has been derived that determines how weak compressibility affects the speed of the incompressible staggered point vortex streets introduced as a model of vortex wakes by von Kármán $[21,22]$. The sign change in the first order relative change in the speed of the street is of particular interest. This means that whether compressibility speeds up or slows down, a given incompressible staggered vortex street is a function of its aspect ratio and it is found that both scenarios are possible. A critical aspect ratio of 0.38187 has been identified at which transition between these two cases occurs. Small aspect ratio compressible streets travel faster than their incompressible counterparts.

Symmetric or unstaggered streets, on the other hand, exhibit no such change in sign in the first-order relative speed correction; they always speed up due to weak compressibility. The effect is maximal for aspect ratios around $\kappa=0.36216$. The explicit formula (61) gives this relative speed correction.

As discussed by Leppington [18], if wavelike problems are of interest, the analysis of the intermediate region presented here can be fed into a full calculation of the global flow by matching of this Rayleigh-Jansen solution to a far-field solution at large distances - where the wavelike nature of the compressible flow must be accounted for-and an inner core region near the vortex. This basic theoretical study may also help in understanding compressible wake structures behind bluff bodies where it has recently been indicated that planar models can play a role [7]. A consequence of our analysis is that the aspect ratio 0.28056 , which is the isolated value for neutral stability in the incompressible case, is well below the critical value of 0.38187 , meaning that making the fluid weakly compressible will speed up a street of small vortices with aspect ratio close to this physically important value. This result is not without interest since it is not clear how one might have predicted this a priori based on current knowledge: While the geometry is clearly very different, the propagation speed of a weakly compressible two-dimensional vortex pair is known to be unchanged to first order in (squared) Mach number [18], while a small-cored axisymmetric vortex ring has been shown by Moore [15] to be slowed down by weak compressibility (the latter being true for Mach numbers that are not necessarily small). Based on the evidence of this paper, we infer that, even for small Mach numbers, the effect of compressibility on global properties of a given vortex equilibrium is a delicate function of its geometry and there appears to be no simple "rule of thumb" that can be generally invoked to make deductions.

The methodology developed here can be applied to other point vortex equilibria to assess the effects of weak compressibility. An important contribution of this paper is to clarify the nature of the force-free condition that must be imposed on a free compressible point vortex in equilibrium.

Leppington's treatment of the compressible point vortex pair [18] was prompted by an earlier numerical study by Moore and Pullin [16], who looked at compressibility effects, at general Mach numbers, on the analytical solutions for a finite-area incompressible hollow vortex pair found by Pocklington [30] (see also recent work by Crowdy et al. [40], where Pocklington's solutions have been reappraised and their linear stability investigated). Crowdy and Green [39] have found that analytical solutions also exist for vortex streets, both staggered and unstaggered, made up of finite-area hollow vortices that constitute a desingularization of von Kármán's vortex streets in exactly the same way that Pocklington's solution provide a desingularization of the point vortex pair. It is clearly of interest to perform a Rayleigh-Jansen analysis for small Mach numbers about the hollow vortex street solutions of Crowdy and Green [39] to gain insights into how the finite size and structure of the vortex cores can affect the speed of such steady compressible streets. The results, 
which require much more detailed consideration of the vortex core structure than needed here for small vortices, will be presented elsewhere [41].

\section{ACKNOWLEDGMENTS}

The authors thank S. Llewellyn Smith for useful discussions. D.G.C. (orcid.org/0000-0002-71620181) is supported by an EPSRC Established Career Fellowship (EP/K019430/10) and a Royal Society Wolfson Research Merit Award. V.S.K. (orcid.org/0000-0002-1518-0994) acknowledges financial support from a scholarship from Imperial College London with additional support from its Department of Mathematics.

\section{APPENDIX: BLASIUS THEOREM FOR WEAKLY COMPRESSIBLE FLOWS}

In this Appendix, we review the extension of the Blasius theorem to weakly compressible flows and derive fresh expressions for the first-order force on an object due to weak compressibility. A similar calculation was carried out in Barsony-Nagy [33]. We want to manipulate the Bernoulli equation (14) to derive an expression for the pressure $p(z, \bar{z})$ in terms of the velocity $\xi(z, \bar{z})$. The Rayleigh-Jansen expansion for the pressure $p(z, \bar{z})$ is taken to be

$$
p(z, \bar{z})=p_{0}(z, \bar{z})+M^{2} p_{1}(z, \bar{z})+o\left(M^{2}\right),
$$

where $p_{0}(z, \bar{z})$ is the incompressible pressure and $p_{1}(z, \bar{z})$ is the first-order weakly compressible correction to be found. Equations (6) and (20) imply that

$$
p=k v^{\gamma}=k v_{s}^{\gamma}\left(\frac{v}{v_{s}}\right)^{\gamma}=\frac{v_{s} c_{s}^{2}}{\gamma}\left(1-\frac{\gamma-1}{2}|\xi|^{2} \frac{M^{2}}{U_{0}^{2}}\right)^{\gamma / \gamma-1} .
$$

This can be expanded for small $M$ as

$$
p=\frac{U_{0}^{2} v_{s}}{\gamma M^{2}}\left(1-\frac{\gamma M^{2}}{2 U_{0}^{2}}\left(\left|\xi_{0}\right|^{2}+M^{2}\left(\xi_{0} \overline{\xi_{1}}+\overline{\xi_{0}} \xi_{1}\right)\right)+M^{4} \frac{\left|\xi_{0}\right|^{4} \gamma}{8 U_{0}^{4}}+o\left(M^{4}\right)\right)
$$

which, on comparison with (A1), yields

$$
p_{0}(z, \bar{z})=\text { constant }-\frac{v_{s}\left|\xi_{0}\right|^{2}}{2} .
$$

This is the familiar incompressible Bernoulli equation. At order $M^{2}$, we find

$$
p_{1}(z, \bar{z})=\frac{v_{s}}{8} \frac{\left|\xi_{0}(z)\right|^{4}}{U_{0}^{2}}-\frac{v_{s}}{2}\left[\xi_{0}(z) \overline{\xi_{1}(z, \bar{z})}+\overline{\xi_{0}(z)} \xi_{1}(z, \bar{z})\right] .
$$

The force, $X-\mathrm{i} Y$, due to the pressure field $p(z, \bar{z})$ on the fluid, or object, contained within a closed streamline $\mathcal{C}$, is [34]

$$
X-i Y=-i \oint_{\mathcal{C}} p(z, \bar{z}) d \bar{z}=\left(X_{0}-i Y_{0}\right)+M^{2}\left(X_{1}-i Y_{1}\right)+o\left(M^{2}\right)
$$

where

$$
X_{0}-i Y_{0}=-i \oint_{\mathcal{C}} p_{0}(z, \bar{z}) d \bar{z}, \quad X_{1}-i Y_{1}=-i \oint_{\mathcal{C}} p_{1}(z, \bar{z}) d \bar{z}
$$

On use of (A4) and the fact that $\mathcal{C}$ is a streamline, which implies $\xi_{0}(z) d z=\overline{\xi_{0}(z)} d \bar{z}$ on $\mathcal{C}$,

$$
X_{0}-i Y_{0}=\frac{i v_{s}}{2} \oint_{\mathcal{C}}\left[\xi_{0}(z)\right]^{2} d z
$$


which is the incompressible Blasius theorem [34]. To find $X_{1}-i Y_{1}$, we use the expression (A5) for $p_{1}(z, \bar{z})$. Since $\mathcal{C}$ is a streamline, it follows that $\xi(z, \bar{z}) d z=\overline{\xi(z, \bar{z})} d \bar{z}$ on $\mathcal{C}$, from which one easily deduces that $\xi_{1}(z, \bar{z}) d z=\overline{\xi_{1}(z, \bar{z})} d \bar{z}$ on $\mathcal{C}$ and, in turn,

$$
\oint_{\mathcal{C}}\left[\xi_{0}(z) \overline{\xi_{1}(z, \bar{z})}+\overline{\xi_{0}(z)} \xi_{1}(z, \bar{z})\right] d \bar{z}=2 \oint_{\mathcal{C}} \xi_{0}(z) \xi_{1}(z, \bar{z}) d z
$$

Therefore,

$$
X_{1}-i Y_{1}=-\frac{i v_{s}}{8 U_{0}^{2}} \oint_{\mathcal{C}}\left|\xi_{0}(z)\right|^{4} d \bar{z}+i v_{s} \oint_{\mathcal{C}} \xi_{0}(z) \xi_{1}(z, \bar{z}) d z
$$

An alternative expression is obtained by substituting for the velocity $\xi_{1}(z, \bar{z})$ from (28) and using the property $\xi_{0}(z) d z=\overline{\xi_{0}(z)} d \bar{z}$ on $\mathcal{C}$ :

$$
\oint_{\mathcal{C}} \xi_{0}(z) \xi_{1}(z, \bar{z}) d z=\frac{1}{4 U_{0}^{2}} \oint_{\mathcal{C}}\left|\xi_{0}(z)\right|^{4} d \bar{z}+\oint_{\mathcal{C}} \xi_{0}(z)\left[\frac{\xi_{0}^{\prime}(z) \overline{I(z)}}{4 U_{0}^{2}}+G^{\prime}(z)\right] d z .
$$

On substitution into (A10), and using the definition of the Imai-Lamla integral (24) to write $d \overline{I(z)}=$ ${\overline{\xi_{0}}(z)}^{2} d \bar{z}$, we arrive at the final expression

$$
X_{1}-i Y_{1}=\frac{i v_{s}}{4 U_{0}^{2}} \oint_{\mathcal{C}} d\left\{\frac{\left[\xi_{0}(z)\right]^{2}}{2} \overline{I(z)}\right\}+i v_{s} \oint_{\mathcal{C}} \xi_{0}(z) G^{\prime}(z) d z
$$

This can be used to find the condition that a compressible point vortex at $z=\eta$ is free of force correct to order $M^{2}$.

Suppose the incompressible velocity field is $\xi_{0}(z)$ with the first-order correction to the complex potential $f_{1}(z, \bar{z})$ given by (23). We shift to a local coordinate system near the vortex and define $\epsilon=z-\eta$. The following local expansions can be derived:

$$
\xi_{0}(z)=\frac{a_{1}}{\epsilon}+a_{2} \epsilon+o(\epsilon), \quad I(z)=\frac{b_{1}}{\epsilon}+C+b_{2} \epsilon+o(\epsilon), \quad G(z)=\frac{c_{1}}{\epsilon}+c_{2} \epsilon+o(\epsilon),
$$

where $a_{1}, a_{2}, b_{1}, b_{2}, c_{1}, c_{2}$, and $C$ are constants and where we have used the fact, noted earlier, that the second-order poles in $G(z)$ at the vortex positions are removable. On isolating the terms in a local expansion of $f_{1}(z, \bar{z})$ that are proportional to $1 / \epsilon$ and $\epsilon$, we find

$$
f_{1}(z, \bar{z})=\left(\frac{a_{1} \bar{C}}{4 U_{0}^{2}}+c_{1}\right) \frac{1}{\epsilon}+\left(\frac{a_{2} \bar{C}}{4 U_{0}^{2}}+c_{2}\right) \epsilon+\text { other terms. }
$$

The coefficient of $1 / \epsilon$ in this series must be zero if we require the point vortex position to be fixed; this follows from the fact that

$$
\log (z-(a+\epsilon \hat{a}))=\log (z-a)-\frac{\epsilon \hat{a}}{z-a}+o(\epsilon),
$$

implying that shifting $a$ by some small amount $\epsilon \hat{a}$ will induce a simple pole at $z=a$. Hence,

$$
\frac{c_{1}}{a_{1}}=-\frac{\bar{C}}{4 U_{0}^{2}}
$$

For the point vortex flows of the type we consider here, both $\xi_{0}(z)$ and $I(z)$ turn out to be single-valued functions so the only contribution to $X_{1}-i Y_{1}$ in (A12) comes from the residue of $\xi_{0}(z) G^{\prime}(z)$, which is easily found to be proportional to

$$
c_{2}-a_{2} \frac{c_{1}}{a_{1}}=\frac{a_{2} \bar{C}}{4 U_{0}^{2}}+c_{2},
$$


where, in the second equality, we have used (A16). But the latter quantity is precisely the $\mathcal{O}(\epsilon)$ term in (A14). Thus the force-free condition on the point vortex is equivalent to setting the coefficient of $\epsilon$ in the series expansion (A14) for $f_{1}(z, \bar{z})$ to be equal to zero. Note that this form of the force-free condition requires the coefficient of $1 / \epsilon$ in the local series expansion of $f_{1}(z, \bar{z})$ about the vortex position to be zero, as enforced in (A16).

[1] C. H. K. Williamson, Vortex dynamics in the cylinder wake, Ann. Rev. Fluid Mech. 28, 477 (1996).

[2] S. K. Lele, Compressibility effects on turbulence, Ann. Rev. Fluid Mech. 26, 211 (1994).

[3] P. G. Saffman, Vortex Dynamics (Cambridge University Press, Cambridge, UK, 1992).

[4] P. K. Newton, The $N$-Vortex Problem: Analytical Techniques (Springer-Verlag, New York, 2001).

[5] J.-Z. Wu, H.-Y. Ma, and M. D. Zhou, Vorticity and Vortex Dynamics (Springer-Verlag, New York, 2006).

[6] D. Bershader, Compressible vortices, in Fluid Dynamics and Its Applications, edited by S. Green (1995), Vol. 30, p. 291.

[7] J.-P. Hickey, F. Hussain, and X. Wu, Compressibility effects on the structural evolution of transitional high-speed planar wakes, J. Fluid Mech. 796, 5 (2016).

[8] S. Mowlavi, C. Arratia, and F. Gallaire, Spatio-temporal stability of the Kármán vortex street and the effect of confinement, J. Fluid Mech. 795, 187 (2016).

[9] M. S. Howe, Theory of Vortex Sound, Cambridge Texts in Applied Mathematics (Cambridge University Press, Cambridge, UK, 2002).

[10] T. Colonius, S. Lele, and P. Moin, The scattering of sound waves by a vortex: Numerical simulations and analytical solutions, J. Fluid Mech. 260, 271 (1994).

[11] R. Ford and S. G. Llewellyn Smith, Scattering of acoustic waves by a vortex, J. Fluid Mech. 386, 305 (1999).

[12] T. Colonius, S. Lele, and P. Moin, The free compressible viscous vortex, J. Fluid Mech. 230, 45 (1991).

[13] K. D. von EllenRieder and B. J. Cantwell, Self-similar, slightly compressible, free vortices, J. Fluid Mech. 423, 293 (2000).

[14] M. J. Mandella, Experimental and analytical studies of compressible vortices, Ph.D. thesis, Dept. of Applied Physics, Stanford University, Stanford, CA, 1987 (unpublished).

[15] D. W. Moore, The effect of compressibility on the speed of a vortex ring, Proc. Roy. Soc. London, Ser. A 397, 87 (1985).

[16] D. W. Moore and D. I. Pullin, The compressible vortex pair, J. Fluid Mech. 185, 171 (1987).

[17] A. Barsony-Nagy, J. Er-El, and S. Yungster, Compressible flow past a contour and stationary vortices, J. Fluid Mech. 178, 367 (1987).

[18] F. G. Leppington, The field due to a pair of line vortices in a compressible fluid, J. Fluid Mech. 559, 45 (2006).

[19] K. Ardalan, D. Meiron, and D. Pullin, Steady compressible vortex flows: The hollow-core vortex array, J. Fluid Mech. 301, 1 (1995).

[20] G. K. O’Reilly and D. I. Pullin, Smooth transonic flow in an array of counter-rotating vortices, J. Fluid Mech. 524, 197 (2005).

[21] T. von Kármán and H. L. Rubach, On the mechanisms of fluid resistance, Physik Z. 13, 49 (1912).

[22] T. von Kármán, Collected works of Theodore von Kármán (Butterworths, London, 1956), Vol. 1.

[23] P. G. Saffman and J. C. Schatzman, Stability of a vortex street of finite vortices, J. Fluid Mech. 117, 171 (1982).

[24] D. I. Meiron, P. G. Saffman, and J. C. Schatzman, The linear two-dimensional stability of inviscid vortex streets of finite-cored vortices, J. Fluid Mech. 147, 187 (1984).

[25] S. Kida, Stabilizing effects of finite core on Kármán vortex street, J. Fluid Mech. 122, 487 (1982).

[26] J. Jiménez, On the linear stability of the inviscid Kármán vortex street, J. Fluid Mech. 178, 177 (1987).

[27] R. von Mises, Mathematical Theory of Compressible Fluid Flow (Dover, New York, 2004). 


\section{SPEED OF A VON KÁRMÁN POINT VORTEX STREET IN ...}

[28] S.-I. Pai, Introduction to the Theory of Compressible Flow (D. Van Nostrand, Princeton, NJ, 1959).

[29] C. Jacob, Introduction Mathématique a la Mécanique des Fluides (Gauthier-Villars, Paris, 1959).

[30] H. C. Pocklington, The configuration of a pair of equal and opposite hollow straight vortices, of finite cross-section, moving steadily through fluid, Proc. Camb. Phil. Soc. 8, 178 (1895).

[31] G. I. Taylor, Recent work on the flow of compressible fluids, J. Lond. Math. Soc. s1-5, 224 (1930).

[32] S. G. Llewellyn Smith, How do singularities move in potential flow? Phys. D (Amsterdam, Neth.) 240, 1644 (2011).

[33] A. Barsony-Nagy, Extension of the Blasius force theorem to subsonic speeds, AIAA J. 23, 1811 (1985).

[34] L. M. Milne-Thomson, Theoretical Hydrodynamics (Dover, New York, 2013).

[35] I. Imai, On the flow of a compressible fluid past a circular cylinder, Proc. Phys. Math. Soc. Jpn. 32, 636 (1938).

[36] I. Imai, On the flow of a compressible fluid past a circular cylinder, II, Proc. Phys. Math. Soc. Jpn. 23, 180 (1941).

[37] I. Imai, A new method of successive approximations for dealing with the two-dimensional subsonic flow of a compressible fluid, Proc. Phys. Math. Soc. Jpn. 24, 120 (1942).

[38] P. G. Saffman and J. C. Schatzman, Properties of a vortex street of finite vortices, SIAM J. Sci. Stat. Comput. 2, 285 (1981).

[39] D. G. Crowdy and C. C. Green, Analytical solutions for von Kármán streets of hollow vortices, Phys. Fluids 23, 126602 (2011).

[40] D. G. Crowdy, S. G. Llewellyn Smith, and D. Freilich, Translating hollow vortex pairs, Eur. J. Mech B/Fluids 37, 180 (2012).

[41] D. G. Crowdy and V. S. Krishnamurthy, The effect of core size on the speed of compressible hollow vortex streets, J. Fluid Mech. (submitted). 